\title{
CONSTITUINTES QUÍMICOS DE Ipomoea subincana MEISN. (CONVOLVULACEAE)
}

Marilena Meira e Jorge M. David

Instituto de Química, Universidade Federal da Bahia, 40170-290 Salvador - BA, Brasil

Juceni P. David ${ }^{*}$, Sinara V. Araújo e Tatiara L. Regis

Faculdade de Farmácia, Universidade Federal da Bahia, 40170-290 Salvador - BA, Brasil

Ana Maria Giulietti e Luciano P. de Queiróz

Departamento de Ciências Biológicas, Universidade Estadual de Feira de Santana, 44031-460 Feira de Santana - BA, Brasil

Recebido em 14/11/07; aceito em 7/3/08; publicado na web em 9/4/08

\begin{abstract}
CHEMICAL CONSTITUENTS OF Ipomoea subincana MEISN. (CONVOLVULACEAE). The chloroform extract of aerial parts of Ipomoea subincana was submitted to different chromatographic procedures which afforded methyl caffeate, ethyl caffeate, methyl 3,4-dimethoxycinnamate, lupeol, $\alpha$-amyrin, $\beta$-amyrin, 3- $\beta$-O- $\beta$-D-glycopiranosyl-sitosterol, $\beta$-sitosterol, stigmasterol, scopoletin, aromadendrane-4 $\beta, 10 \alpha$-diol, $n$-docosyl-cis-p-coumarate and $n$-icosyl-trans- $p$-coumarate, vanilin, cinamic acid and vanillic acid. However, from the ethyl acetate extract besides quercetin and 3-O- $\beta$-D-glycopiranosyl-quercetin were isolated methyl 4-O- $E$-feruloyl5-O-E-caffeoyl-quinate, methyl 3,5-di-O-E-caffeoyl-quinate and methyl 4-O-E-caffeoyl-quinate. The structures of the compounds were established on the basis of spectral data.
\end{abstract}

Keywords: Ipomoea subincana; caffeoyl quinates; aromadendrane-4 $4,10 \alpha$-diol.

\section{INTRODUÇÃo}

A família Convolvulaceae é formada por 57 gêneros contendo cerca de 1600 espécies. O gênero Ipomoea é o mais representativo da família, compreendendo cerca de 700 espécies que medram nas regiões tropicais e temperadas do globo. Na região do semi-árido do Nordeste brasileiro foram registradas 47 espécies distribuídas em 10 gêneros, sendo que o gênero Ipomoea está representado por 7 espécies. ${ }^{1}$ De modo geral, as espécies de Ipomoea são utilizadas na medicina popular no tratamento das mais diversas enfermidades, tais como, reumatismo, artrite, hipertensão, furúnculos, doenças renais, desordens digestivas e disenterias. ${ }^{2}$ Em estudos anteriores com algumas espécies do gênero Ipomoea foram observadas diversas atividades biológicas, entre estas, insulinogênica, hipoglicêmica e anticancerígena.,

Ipomoea subincana Meisn. é uma planta endêmica do semiárido brasileiro sobre a qual, até o presente, não constam relatos de estudos químicos ou farmacológicos na literatura. Dessa forma, este trabalho descreve os resultados obtidos no estudo fitoquímico das partes aéreas de um espécime de I. subincana, coletado na localidade de Morro do Chapéu, Bahia. A partir do extrato clorofórmico foram isolados cafeato de metila, cafeato de etila, 3,4-dimetoxi-cinamato de metila, lupeol, $\alpha$-amirina, $\beta$-amirina, $\beta$ sitosterol, 3- $\beta$-O- $\beta$-D-glicopiranosil-sitosterol, estigmasterol, escopoletina, aromadendrane-4 $\beta, 10 \alpha$-diol (1), cis $p$-cumarato de $\mathrm{n}$-docosila, trans p-cumarato de $\mathrm{n}$-icosila, vanilina, ácido cinâmico e ácido vanílico. A partir do fracionamento do extrato acetato de etila foram isolados quercetina, quercetina-3-O- $\beta$-D-glicopiranosídeo, 4-O-E-feruloil-5-O-E-cafeoil-quinato de metila (2), 3,5-di-O-E-cafeoil-quinato de metila (3) e 4-O-E-cafeoil-quinato de metila (4). A identificação estrutural das substâncias foi realizada através da análise de dados espectrométricos, tais como, EMIE, $\mathrm{RMN}$ de ${ }^{1} \mathrm{He}{ }^{13} \mathrm{C}$ (BB e DEPT).

\footnotetext{
*e-mail: juceni@ufba.br
}

\section{RESULTADOS E DISCUSSÃO}

Os triterpenos (lupeol, $\alpha$-amirina, $\beta$-amirina), ${ }^{5,6}$ esteróides (3$\beta$-O- $\beta$-glicopiranosil-sitosterol, $\beta$-sitosterol, estigmasterol), ${ }^{7}$ cis$p$-cumarato de n-docosila e trans- $p$-cumarato de n-icosila, ${ }^{8}$ os ácidos cinâmico ${ }^{9}$ e vanílico, ${ }^{10}$ vanilina, ${ }^{10}$ cafeato de metila, ${ }^{11}$ cafeato de etila, ${ }^{11}$ escopoletina, ${ }^{12}$ aromadendrano-4 $\beta, 10 \alpha$-diol, ${ }^{13} 3,4$ dimetoxi-cinamato de metila, ${ }^{14}$ bem como os flavonóides (quercetina, quercetina 3-O- $\beta$-D-glicopiranosídeo) ${ }^{15}$ foram identificados através da comparação direta dos dados espectroscópicos obtidos com os descritos na literatura para essas substâncias.

A substância 1 apresentou o pico do íon molecular em $\mathrm{m} / z 238$ no espectro de massas que, aliado aos dados obtidos dos espectros de $\mathrm{RMN}{ }^{13} \mathrm{C}\left(\mathrm{BB}, \mathrm{DEPT} 135^{\circ}\right.$ e $90^{\circ}$ ) permitiu sugerir a fórmula molecular $\mathrm{C}_{15} \mathrm{H}_{26} \mathrm{O}_{2}$. A análise dos dados de RMN demonstrou que a substância $\mathbf{1}$ apresentava quatro sinais metínicos, quatro metilênicos, quatro metílicos e três sinais de carbonos não hidrogenados, sendo que dois desses últimos eram oxigenados. $\mathrm{O}$ espectro RMN ${ }^{1} \mathrm{H}$ mostrou a presença de sinais apenas na região entre $\delta 0,4$ e $\delta$ 1,9. Essas informações obtidas nos espectros de RMN em conjunto com a fórmula molecular permitiram reconhecer o esqueleto de um sesquiterpeno para a substância. $\mathrm{O}$ espectro de $\mathrm{RMN}{ }^{1} \mathrm{H}$ mostrou ainda a presença de sinais característicos de anel ciclopropânico em $\delta 0,44(d d ; 9$ e 9,6$)$ e $\delta 0,67(m)$, possibilitando caracterizar o esqueleto aromadendrano. ${ }^{13}$ A comparação dos dados de RMN ${ }^{13} \mathrm{C}$ com valores constantes na literatura ${ }^{13}$ mostraram que a substância 1 (Figura 1) corresponde ao aromadendrano- $4 \beta, 10 \alpha$-diol. ${ }^{13}$ Tanto a estrutura quanto a atribuição dos dados de RMN foram confirmadas pela análise dos espectros HMQC e HMBC.

O espectro RMN ${ }^{1} \mathrm{H}$ da substância 2 mostrou sinais para 4 dubletos em $\delta 7,66, \delta 6,40, \delta 7,50$ e $\delta 6,17$ (Tabela 1) característicos de hidrogênios olefínicos em configuração trans $(\mathrm{J}=15,9 \mathrm{~Hz})$, além de sinais correspondentes a dois sistemas de spins do tipo AMX indicando a presença de dois anéis aromáticos 1,3,4-tri-substituídos (Tabela 1). No espectro de RMN ${ }^{1} \mathrm{H}$ pôde ser observada ainda a presença de um multipleto entre $\delta$ 2,06-2,40 atribuído aos hidrogênicos metilênicos 


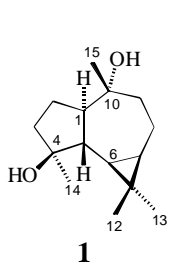<smiles>COc1cc(/C=C/C(=O)OC2CC(O)(C(C)=O)CC(O)C2OC(=O)CCc2ccc(O)c(O)c2)ccc1O</smiles><smiles>CC(=O)OC1(O)CC(OC(=O)C=Cc2ccc(O)c(O)c2)C(O)C(OC(=O)C=Cc2ccc(O)c(O)c2)C1</smiles><smiles>CC(=O)OC1(O)CC(O)C(OC(=O)C=Cc2ccc(O)c(O)c2)C(O)C1</smiles>

Figura 1. Substâncias isoladas de I. subincana

(H-2 e H-6) da unidade quinato, além da presença de dois grupos metoxílicos $(\delta 3,72$ e $\delta 3,85)$. Porém, as informações mais importantes neste espectro foram aquelas referentes aos três hidrogênios oximetínicos, uma vez que os efeitos de desproteção observados para H-4 $(\delta 5,13)$ e H-5 $(\delta 5,50)$ quando comparados ao H-3 $(\delta 4,40)$ indicaram que as unidades cafeoila e feruloila estavam esterificando as hidroxilas das posições 4 e 5 do ácido quínico. O espectro HMBC confirmou que a carboxila do ácido quínico se encontrava esterificada com um grupo metoxila através da correlação entre o singleto em $\delta$ 3,72 e o sinal em $\delta 175,2$ (C-7) permitindo, assim, atribuir o valor de $\mathrm{RMN}$ de ${ }^{1} \mathrm{H}$ para o grupo metoxílico e de $\mathrm{RMN}$ de ${ }^{13} \mathrm{C}$ para a carboxila. A análise deste espectro permitiu também confirmar a presença da unidade feruloil através da correlação entre o sinal $\delta 3,85$ com o carbono em $\delta 149,3$ (C-3') e estabelecer que tanto C-4 quanto C-5 estavam esterificados, respectivamente, com os ácidos ferúlico e cafeico. Esta última informação pode ser deduzida através das correlações observadas entre os sinais em $\delta 5,13(\mathrm{H}-4)$ e $\delta 5,50(\mathrm{H}-5)$ com as carbonilas C-9' $(\delta 168,4)$ e C-9"' $(\delta 167,9)$, respectivamente. A comparação dos dados de $\mathrm{RMN}{ }^{1} \mathrm{H}$ da substância $\mathbf{2}$ com os valores constantes na literatura ${ }^{16}$ permitiu concluir que esta substância corresponde a 4-O- $E$-feruloil-5-O- $E$-cafeoil-quinato de metila (2).

Os espectros EM-APCI das substâncias 3 e 4 sugeriam as fórmulas moleculares $\mathrm{C}_{26} \mathrm{H}_{26} \mathrm{O}_{12}$ e $\mathrm{C}_{17} \mathrm{H}_{20} \mathrm{O}_{9}$ através dos íons pseudomoleculares $m / z, 529[\mathrm{M}-\mathrm{H}]^{+}$e $\mathrm{m} / z$ de $367[\mathrm{M}-\mathrm{H}]^{+}$, respectivamente. Aliado a isso, os espectros de $\mathrm{RMN}{ }^{1} \mathrm{H}$ e $\mathrm{RMN}{ }^{13} \mathrm{C}$ de 3 e 4 evidenciaram somente a presença das unidades cafeoil e quinato de metila. A análise destes dados (Tabelas 2 e 3) permitiu sugerir a presença de duas unidades cafeoilas na substância 3. Por outro lado, a presença da unidade quinato de metila foi sugerida por ambos os espectros RMN $\left({ }^{13} \mathrm{C}\right.$ e $\left.{ }^{1} \mathrm{H}\right)$ através dos sinais de metoxila e de três carbonos oximetínicos $(\delta 72,2, \delta 71,9$ e $\delta 69,0)$. No espectro de RMN de ${ }^{1} \mathrm{H}$ os hidrogênios oximetínicos H-3 $(\delta 5,40)$ e H-5 $(\delta 5,30)$ encontravam-se mais desprotegidos quando comparados com $\mathrm{H}-4$ $(\delta 3,98)$, sugerindo que as unidades cafeoilas estavam esterificando as hidroxilas das posições 3 e 5 do quinato de metila. $\mathrm{O}$ espectro HMBC comprovou que a unidade do ácido quínico era um derivado metilado através da correlação registrada entre o singleto em $\delta 3,69$ com a carbonila C-7. Este espectro permitiu também comprovar que a existência de esterificação em C-3 e C-5 devido às correlações observadas entre $\delta 5,40(\mathrm{H}-3)$ e $\delta 5,30(\mathrm{H}-5)$ com as carbonilas C-9'
Tabela 1. Dados de RMN da substância $2\left(\mathrm{CD}_{3} \mathrm{OD}\right)$

\begin{tabular}{|c|c|c|c|}
\hline & $\mathrm{H}(\delta)$ & $\mathrm{C}(\delta)(\mathrm{HMQC})$ & (HMBC) \\
\hline 1 & - & 75,7 & - \\
\hline 2 & $2,06-2,40(m)$ & 38,3 & $\begin{array}{c}175,2 ; 75,7 \\
68,6 ; 74,8\end{array}$ \\
\hline 3 & $4,40(d d d ; 3,0 ; 6,0$ e 3,0$)$ & 68,6 & 75,7 \\
\hline 4 & $5,13(d d ; 3,0$ e 8,1$)$ & 74,8 & 168,4 \\
\hline 5 & $5,50(d d d ; 5,4 ; 7,8$ e 8,1$)$ & 69,0 & 74,$8 ; 167,9$ \\
\hline 6 & $2,06-2,40(m)$ & 38,3 & $\begin{array}{l}69,0 ; 75,7 ; \\
74,8 ; 175,2\end{array}$ \\
\hline 7 & - & 175,2 & - \\
\hline $\mathrm{OCH}_{3}$ & $3,72(s)$ & 53,1 & 175,2 \\
\hline $1^{\prime}$ & - & 127,5 & - \\
\hline $2^{\prime}$ & $7,15(d ; 1,5)$ & 111,6 & 124,$2 ; 150,7$ \\
\hline $3^{\prime}$ & - & 149,3 & - \\
\hline $4^{\prime}$ & - & 150,6 & - \\
\hline $5^{\prime}$ & $6,78(d ; 9,0)$ & 116,5 & 127,$5 ; 149,3$ \\
\hline $6^{\prime}$ & $7,05(d d ; 9,0$ e 1,5$)$ & 124,2 & 111,6 \\
\hline $7^{\prime}$ & $7,66(d ; 15,9)$ & 147,6 & $\begin{array}{c}124,2 ; 111,6 \\
168,4\end{array}$ \\
\hline $8^{\prime}$ & $6,40(d ; 15,9)$ & 115,1 & 127,5 \\
\hline $9^{\prime}$ & - & 168,4 & - \\
\hline $\mathrm{OCH}_{3}$ & $3,85(s)$ & 56,3 & 149,3 \\
\hline $1 "$ & - & 127,6 & - \\
\hline $2^{\prime \prime}$ & $7,01(d ; 1,8)$ & 115,1 & 123,$1 ; 149,8$ \\
\hline $3 "$ & - & 146,8 & - \\
\hline $4 "$ & - & 149,8 & - \\
\hline $5 "$ & $6,78(d ; 9,0)$ & 116,4 & 127,$6 ; 146,8$ \\
\hline $6 "$ & $6,92(d d ; 8,4$ e 1,5$)$ & 123,1 & 115,1 \\
\hline 7" & $7,50(d ; 15,9)$ & 147,7 & $\begin{array}{c}123,1 ; 115,1 ; \\
167,9\end{array}$ \\
\hline $8 "$ & $6,17(d ; 15,9)$ & 114,5 & 127,6 \\
\hline $9 "$ & - & 167,9 & - \\
\hline
\end{tabular}

$(\delta 168,7)$ e C-9" ( $\delta 167,9)$, respectivamente. A comparação dos dados de RMN de ${ }^{1} \mathrm{H}$ e ${ }^{13} \mathrm{C}$ da substância 3 com dados de modelos constantes na literatura ${ }^{11,17}$ permitiram concluir que essa substância corresponde ao 3,5-di-O-E-cafeoil-quinato de metila.

$\mathrm{O}$ conjunto de sinais nos espectros RMN $\left(\mathrm{He} \mathrm{e}^{13} \mathrm{C}\right)$ de $\mathbf{4}$ indicou a presença de um derivado do ácido quínico esterificado com apenas uma unidade cafeoila (Tabelas 2 e 3). Este fato foi evidenciado pelo espectro de $\mathrm{RMN}$ de ${ }^{1} \mathrm{H}$ que mostrou sinal para apenas um hidrogênio oximetínico mais desprotegido (H-4). O deslocamento químico observado para $\mathrm{H}-4(\delta 5,11)$ quando comparado aos do H-5 ( $\delta 4,30)$ e H-3 $(\delta 4,00)$ mostrou que este estava sob efeito de desproteção, sugerindo que a unidade cafeoila estava esterificando somente a hidroxila da posição 4 da unidade quinato de metila. Essa observação foi confirmada a partir da correlação registrada no espectro HMBC (Tabela 3) entre o sinal do H-4 e a carboxila (C-9'). Além disso, o espectro HMBC confirmou que o ácido quínico estava esterificado com um grupo metoxila, através da correlação entre $\delta 3,72$ e $\delta 175,2$ (C-7). A comparação dos dados de RMN ${ }^{1} \mathrm{H}$ da substância 4 com os de valores constantes na literatura ${ }^{18}$ para o ácido 4-O-E-cafeoilquínico permitiu concluir que esta substância corresponde ao 4-O-E-cafeoil-quinato de metila.

Esta é a primeira vez que o sesquiterpeno aromadendrane$4 \delta, 10 \alpha$-diol (1) foi isolado na família Convolvulaceae. Essa substância que apresenta atividade antifúngica ${ }^{13}$ foi previamente obtida de Xylopia brasiliensis (Anonaceae). As substâncias $\mathbf{2}$ e 4 foram isoladas pela primeira vez na família Convolvulaceae. Já a substância 3, foi isolada anteriormente de I. pescaprae e apresenta ati- 
Tabela 2. Dados de RMN da substância $3\left(\mathrm{CD}_{3} \mathrm{OD}\right)$

\begin{tabular}{|c|c|c|c|}
\hline & $\mathrm{H}(\delta)$ & $\mathrm{C}(\delta)(\mathrm{HMQC})$ & (HMBC) \\
\hline 1 & - & 74,6 & - \\
\hline 2 & $2,30-2,19(m)$ & 38,4 & $\begin{array}{c}175,6 ; 74,6 \\
71,9 ; 69,1\end{array}$ \\
\hline 3 & $5,40(m)$ & 71,9 & 168,$7 ; 74,6$ \\
\hline 4 & $3,98(d d ; 3,3$ e 9,3$)$ & 69,1 & 72,$2 ; 71,9$ \\
\hline 5 & $5,30(m)$ & 72,2 & 167,9 \\
\hline 6 & $2,30-2,19(m)$ & 35,6 & $\begin{array}{c}175,6 ; 74,6 \\
72,2 ; 69,1\end{array}$ \\
\hline 7 & - & 175,6 & - \\
\hline $\mathrm{OCH}_{3}$ & $3,69(s)$ & 53,0 & 175,6 \\
\hline $1^{\prime}$ & - & 127,5 & - \\
\hline $2^{\prime}$ & $7,07(d ; 2,4)$ & 115,1 & $\begin{array}{c}149,7 ; 146,7 \\
123,1\end{array}$ \\
\hline $3^{\prime}$ & - & 146,7 & - \\
\hline $4^{\prime}$ & - & 149,7 & - \\
\hline $5^{\prime}$ & $6,97(d ; 8,1)$ & 123,1 & $\begin{array}{c}149,7 ; 146,7 \\
115,1\end{array}$ \\
\hline $6^{\prime}$ & $6,79(d d ; 8,1$ e 2,4$)$ & 116,4 & $\begin{array}{c}149,7 \\
146,7 ; 127,5\end{array}$ \\
\hline $7^{\prime}$ & $7,62(d ; 15,9)$ & 147,1 & $\begin{array}{c}168,7 ; 123,1 \\
115,1\end{array}$ \\
\hline $8^{\prime}$ & $6,34(d ; 15,9)$ & 115,4 & 127,$5 ; 168,7$ \\
\hline $9^{\prime}$ & - & 168,7 & - \\
\hline $1 "$ & - & 127,8 & - \\
\hline $2 "$ & $7,07(d ; 2,4)$ & 115,1 & $\begin{array}{c}149,7 ; 146,7 \\
123,1\end{array}$ \\
\hline 3" & - & 146,7 & - \\
\hline $4 "$ & - & 149,7 & - \\
\hline $5 "$ & $6,97(d ; 8,1)$ & 123,1 & $\begin{array}{c}149,7 ; 146,7 \\
115,1\end{array}$ \\
\hline $6 "$ & $6,79(d d ; 8,1$ e 2,4$)$ & 116,5 & $\begin{array}{c}149,7 \\
146,7 ; 127,8\end{array}$ \\
\hline 7" & $7,55(d ; 15,9)$ & 147,4 & $\begin{array}{c}123,1 ; 115,1 \\
167,9\end{array}$ \\
\hline $8 "$ & $6,22(d ; 15,9)$ & 114,8 & 127,$8 ; 167,9$ \\
\hline 9" & - & 167,9 & - \\
\hline
\end{tabular}

Tabela 3. Dados de RMN da substância $4\left(\mathrm{CD}_{3} \mathrm{OD}\right)$

\begin{tabular}{|c|c|c|c|}
\hline & $\mathrm{H}(\delta)$ & C (ס) (HMQC) & (HMBC) \\
\hline 1 & - & 75,8 & - \\
\hline 2 & $2,30-2,19(m)$ & 38,4 & $\begin{array}{c}175,2 ; 75,8 \\
74,8 ; 69,0\end{array}$ \\
\hline 3 & $4,00(\mathrm{~m})$ & 69,0 & - \\
\hline 4 & $5,11(d d ; 3,0$ e 8,1$)$ & 74,8 & $\begin{array}{c}168,5 ; 69,0 \\
38,4\end{array}$ \\
\hline 5 & 4,30 (m) & 68,6 & 69,0 \\
\hline 6 & $2,30-2,19(m)$ & 35,6 & $\begin{array}{c}175,2 ; 74,8 \\
72,2 ; 68,6\end{array}$ \\
\hline 7 & - & 175,2 & - \\
\hline $\mathrm{OCH}_{3}$ & $3,72(s)$ & 53,1 & 175,2 \\
\hline $1^{\prime}$ & - & 127,6 & - \\
\hline $2^{\prime}$ & $7,03(d ; 2,4)$ & 115,1 & $\begin{array}{c}149,6 ; 146,8 ; \\
123,0\end{array}$ \\
\hline $3^{\prime}$ & - & 146,8 & - \\
\hline $4^{\prime}$ & - & 149,6 & - \\
\hline $5^{\prime}$ & $6,97(d ; 8,1)$ & 123,0 & $\begin{array}{c}149,6 ; 146,8 \\
115,1\end{array}$ \\
\hline $6^{\prime}$ & $6,76(d d ; 8,1$ e 2,4$)$ & 116,5 & $\begin{array}{c}149,6 ; 146,8 \\
127,6\end{array}$ \\
\hline $7^{\prime}$ & $7,62(d ; 15,6)$ & 147,7 & $\begin{array}{c}168,5 ; 123,0 \\
115,1\end{array}$ \\
\hline 81 & $6,30(d ; 15,6)$ & 114,7 & 127,$6 ; 168,5$ \\
\hline $9^{\prime}$ & - & 168,5 & - \\
\hline
\end{tabular}

vidade inibitória à síntese da colagenase. ${ }^{19}$ Outros derivados esterificados do ácido quínico já haviam sido isolados anteriormente em I. pescaprae, ${ }^{19}$ I. aquatica, ${ }^{20}$ I. $_{\text {batatas }}{ }^{11}$ e I. fistulosa ${ }^{21} \mathrm{e}$ apresentam diversas atividades biológicas, tais como, inibitória da enzima colagenase, ${ }^{19}$ hipoglicêmica, ${ }^{20}$ inibição da replicação do $\mathrm{HIV}^{22}$ e antifúngica. ${ }^{23}$ Além disso, este trabalho permitiu através de técnicas bidimensionais a atribuição inequívoca dos sinais de RMN de 2, 3 e 4.

\section{PARTE EXPERIMENTAL}

\section{Procedimentos experimentais gerais}

Os pontos de fusão (P.f.) foram determinados em aparelho digital da Microquímica modelo APF-301 e os valores obtidos não foram corrigidos. Os espectros de $\mathrm{RMN}{ }^{1} \mathrm{H} \mathrm{e}{ }^{13} \mathrm{C}$ foram registrados em espectrômetro da Varian, modelo Gemini 2000, operando a 300 MHZ para ${ }^{1} \mathrm{H}$ e $75 \mathrm{MHz}$ para ${ }^{13} \mathrm{C}$. Os deslocamentos químicos foram registrados em $\delta$ ( $\mathrm{ppm}$ ) em referência ao TMS. Os espectros de massas foram obtidos em detector de massas da HP modelo 5973 e em sistema LC/MS da Shimadzu modelo LC 2010 utilizando como eluente $\mathrm{MeOH} / \mathrm{H}_{2} \mathrm{O}$ 9:1 e sistema de ionização atmospheric pressure chemical ionisation (APCI).

\section{Material vegetal}

As partes aéreas de I. subincana foram coletadas na localidade de Morro do Chapéu, em área de caatinga arbustiva sobre solo arenoso, em 9 de maio de 2003. A exsicata do espécime testemunha ( $L$. P. de Queiróz 774) está depositada no Herbário da Universidade Estadual de Feira de Santana, sob o número 71221.

\section{Procedimento para obtenção dos extratos e isolamento dos constituintes}

As partes aéreas (4389 g) de I. subincana foram secas, moídas e maceradas com $\mathrm{MeOH}$. O extrato metanólico $(620 \mathrm{~g})$ foi submetido a sucessivas partições entre $\mathrm{CHCl}_{3}: \mathrm{MeOH} / \mathrm{H}_{2} \mathrm{O}(6: 4) \mathrm{e}$ AcOEt: $\mathrm{H}_{2} \mathrm{O}$ (1:1). O extrato clorofórmico (144,5 g) obtido foi submetido à cromatografia em coluna $(\mathrm{CC})$ sob sílica gel $(\mathrm{CC}$ principal), usando como sistema de solvente hexano:AcOEt e $\mathrm{CHCl}_{3}: \mathrm{MeOH}$ em ordem crescente de polaridade e as frações obtidas foram purificadas como descrito a seguir. A fração da CC principal eluída em hexano:AcOEt 8:2 foi submetida à purificação em outra CC sob sílica gel utilizando como eluente hexano:AcOEt 95:5. Desta coluna foram obtidos trans-p-cumarato de n-icosila $(168,2$ $\mathrm{mg}$ ), cis- $p$-cumarato de n-docosila (20,0 mg), lupeol (23,6 mg), a mistura de lupeol, $\alpha$-amirina e $\beta$-amirina $(185,8 \mathrm{mg})$, bem como, a mistura de $\beta$-sitosterol e estigmasterol (136,6 mg). A fração da CC principal eluída em $\mathrm{CHCl}_{3}: \mathrm{MeOH}$ 9:1 foi purificada por meio $\mathrm{CC}$ utilizando como eluentes hexano:AcOEt 9:1, 8:2 e 6:4, fornecendo as substâncias 3- $\beta$-O- $\beta$-glicopiranosil-sitosterol (152,3 mg), ácido vanílico $(3,2 \mathrm{mg})$, aromadendrano- $4 \beta, 10 \alpha$-diol (1), vanilina $(9,8$ $\mathrm{mg}$ ) e escopoletina $(21,0 \mathrm{mg})$, respectivamente. A fração da CC principal eluída em $\mathrm{CHCl}_{3}: \mathrm{MeOH}$ 8:2 foi submetida à $\mathrm{CC}$ sob sílica gel utilizando AcOEt:MeOH 7:3. Esta fração (7:3) após hidrólise alcalina $(\mathrm{NaOH} 0,1 \mathrm{~N})$ e extração com $\mathrm{CHCl}_{3}$ forneceu ácido cinâmico $(58,0 \mathrm{mg})$. A fração da CC principal eluída em $\mathrm{CHCl}_{3}: \mathrm{MeOH}$ 6:4 foi submetida a nova $\mathrm{CC}$ sob sílica gel utilizando como eluente $\mathrm{CHCl}_{3}: \mathrm{MeOH}$ 95:5 e 9:1 fornecendo 3,4-dimetoxicinamato de metila $(11,2 \mathrm{mg})$, bem como cafeato de metila e cafeato de etila $(5,2 \mathrm{mg})$, respectivamente.

$\mathrm{O}$ extrato acetato de etila $(25,94 \mathrm{~g})$ foi submetido à $\mathrm{CC}$ filtrante 
sob sílica gel, utilizando o sistema de solvente AcOEt/MeOH em gradiente crescente de polaridade. A fração da CC principal eluída em AcOEt/MeOH 9:1 forneceu quercetina (13,0 mg), quercetina 3-O- $\beta$-D-glicopiranosídeo (20,1 mg), após CC sob Si gel utilizando-se como eluente AcOEt/MeOH 9:1. A fração eluída em AcOEt:MeOH 7:3 foi purificada por meio CC sob Si gel utilizando-se como eluente AcOEt:MeOH 9:1 resultando no isolamento de 4-O-E-feruloil-5-O-E-cafeoil-quinato de metila (2; 14,8 mg), 3,5 -di-O-E-cafeoil-quinato de metila $(\mathbf{3} ; 5,1 \mathrm{mg})$ e $4-\mathrm{O}-E$-cafeoilquinato de metila $(4 ; 14,1 \mathrm{mg})$.

\section{Aromadendrano- $4 \beta, 10 \alpha$-diol (1)}

Óleo (10,0 mg). RMN ${ }^{1} \mathrm{H}\left(300 \mathrm{MHz}, \mathrm{CDCl}_{3}\right)$ : 1,87 (1H, $m, \mathrm{H}-$ 1), 1,65 (2H, $m, \mathrm{H}-2), 1,67$ (2H, $m, \mathrm{H}-3), 1,18(1 \mathrm{H}, m, \mathrm{H}-5), 0,44$ $(1 \mathrm{H}, d d, 9,0$ e $9,6, \mathrm{H}-6), 0,67$ (1H, $m, \mathrm{H}-7), 1,81(2 \mathrm{H}, m, \mathrm{H}-8), 1,53$ (1H, $m, \mathrm{H}-9 \mathrm{a}), 1,77$ (1H, $m, \mathrm{H}-9 \mathrm{~b}), 1,04$ (6H, $s, \mathrm{H}-12$ e H-13), 1,25 (3H, $s, \mathrm{H}-14), 1,18$ (3H, $s, \mathrm{H}-15)$. $\mathrm{RMN}{ }^{13} \mathrm{C}$ (75 MHz, $\left.\mathrm{CDCl}_{3}\right): \beta$ 56,4(C-1), 23,8(C-2), 41,1(C-3), 80,4(C-4), 48,4(C-5), 28,3(C-6), 26,6 (C-7), 20,1(C-8), 44,4(C-9), 75,0(C-10), 19,4(C-11), 28,6(C12), 16,4 (C-13), 24,4 (C-14), 20,3(C-15). EMIE (70 eV) $\mathrm{m} / z$ (intensidade relativa): $[\mathrm{M}]+238$ (2), 220 (12), 192 (5), 178 (72), 162 (52), 163 (50), 149 (39), 140 (51), 135 (77), 123 (59), 111 (100), 109 (72), 95(69), 93 (54), 85 (50), 81 (47), 69 (41), 57 (50).

\section{AGRADECIMENTOS}

Ao CNPq, IMSEAR e FAPESB pelas bolsas e auxílio financeiro e à bióloga M. E. Junqueira pela coleta da espécie.

\section{REFERÊNCIAS}

1. Coelho, A. A. O. P. Em Diversidade e caracterização das Fanerógamas do Semi-árido Brasileiro; Giulietti, A. M.; Conceição, A. A.; Queiroz, L. P., eds.; Instituto do Milênio do Semi-árido, Editora APN, 2006, vol. 1, p. 88.

2. Pereda-Miranda, R.; Escalante-Sánchez, E.; Escobedo-Martínez, C.; J. Nat. Prod. 2005, 68, 226

3. Khan, M. M.; Ahmad, F.; Rastogi, A. K.; Kidwai, J. R.; Fitoterapia 1994, 65,231

4. Cao, S.; Guzza, R. C.; Wisse, J. H.; Miller, J. S.; Evans, R.; Kingston, D. G. I.; J. Nat. Prod. 2005, 68, 487.

5. Mahato, S. B.; Kundu, A. P.; Phytochemistry 1994, 37, 1517.

6. Reynolds, W. F.; McLean, S.; Poplawski, J.; Tetrahedron 1986, 42, 3419

7. Correia, S. J.; David, J. P.; David, J. M.; Quim. Nova 2003, 26, 36.

8. Mahmood, U.; Vijay, K. K.; Acharya, R.; Jirovetz, L.; Phytochemistry 2003, $64,851$.

9. Kalinowska, M.; ZwisBocka, R.; Lewandowski, W.; J. Mol. Struct. 2007, $834,572$.

10. Harrison, L. J.; Sia, G.-L.; Sim, K.-Y.; Tan, H. T.-W.; Connolly, J. D.; Lavaui, C.; Massiot, G.; Phytochemistry 1995, 38, 1497.

11. Islam, M. S.; Yoshimoto, M.; Yahara, S.; Okuno, S.; Ishiguro, K. Yamakawa, O.; J. Agric. Food Chem. 2002, 50, 3718.

12. Vasconcelos, J. M. J.; Silva, A. M. S.; Cavaleiro, J. A. S.; Phytochemistry 1998, 49, 1421.

13. Moreira, C.; Lago, J. H. G.; Young, M. C. M.; Roque, N. F.; J. Braz. Chem. Soc. 2003, 14, 828 .

14. Forrest, T. P.; Ray, S.; Phytochemistry 1972, 11, 855.

15. Bennini, B.; Chulia, A. J.; Kaouadji, M.; Thomasson, F.; Phytochemistry 1992, 31, 2483.

16. Nogata, Y.; Sekiya, K.; Ohta, H.; Kusumoto, K.; Ishizu, T.; Phytochemistry 2001, 56, 729 .

17. Chuda, Y.; Ono, H.; Ohnishi-Kameyaa, M.; Nagata, T.; Tsushida, T.; J. Agric. Food Chem. 1996, 44, 2037.

18. Dini, I.; Tenore, G. C.; Dini, A.; J. Agric. Food Chem. 2006, 54, 8733.

19. Teramachi, F.; Koyano, T.; Kowithayakor, T.; Hayashi, M.; Komiyama, K.; Ishibashi, M.; J. Nat. Prod. 2005, 68, 794.

20. Okudaira, R.; Kyanbu, H.; Ichiba, T.; Toyokawa, T.; Jpn. Kokai Tokkyo Koho 2005, JP 2005213221.

21. Sattar, E. A.; Gala, A.; Rashwan A.; Int. J. Pharm. 1995, 33, 155.

22. Mahmood, N.; Moore, P. S.; Tommasi, N. D.; Simone, F. D.; Colman, S.; Hay, A. J.; Pizza, C.; Antiviral Chem. Chemother. 1993, 4, 235.

23. Stange Jr, R. R.; Midland, S. L.; Holmes, G. J.; Sims, J. J.; Mayer, R. T.; Postharvest Biol. Tec. 2001, 23, 85. 